\title{
Determinación inmunohistoquímica y utilidad pronóstica del receptor del factor de crecimiento epidérmico en los tumores estromales gastrointestinales
}

\author{
D. Padilla, P. Menéndez, M. García ${ }^{1}$, P. Villarejo, T. Cubo, D. Gambí, R. Pardo y J. Martín \\ Servicios de Cirugía General y de Aparato Digestivo y 'Anatomía Patológica. Hospital General. Ciudad Real
}

\section{RESUMEN}

Introducción: el receptor del factor de crecimiento epidérmico, EGFR(HER-1), es un receptor de tirosina quinasas cuya activación permite un aumento de la proliferación celular, angiogénesis, proceso metastásico y disminución de la apoptosis celular. Nuestro objetivo es conocer el valor pronóstico de la inmunotinción de EGFR en tumores estromales gastrointestinales (GIST).

Pacientes y método: estudio retrospectivo que incluye todos los GIST intervenidos quirúrgicamente entre 1995-2007 en el Servicio de Cirugía General y del Aparato Digestivo del Hospital General de Ciudad Real. Variables clínicas: edad, sexo, clínica, mortalidad, recidiva. Variables patológicas: a) macroscópicas: localización, diámetro; b) microscópicas: necrosis tumoral, índice mitótico, tipo celular; y c) inmunohistoquímicas: vimentina (V9, Dako A/s); actina del músculo liso (HHF-35, Biogenex); CD34 (QBEND/10); S100 (Policlonal Dako A/S); CD117 (c-kit Rabbit, antihuman polyclonal antibody, 1:600); PDGFR-alfa (Rabbit polyclonal antibody, 1:50, Sta. Cruz Biotechnology). Variables moleculares pronósticas: P-53, PAb240 (DakoCytomation), 1:75, Ki-67, clona MIB1 (Dako), 1:120 y EGFR pharmDx ${ }^{\mathrm{TM}}$ Dako Autostainer (Dako, Dinamarca). Criterios de malignidad: criterios de Fletcher.

Resultados: entre 1995 y 2007, 35 GIST, fueron intervenidos quirúrgicamente en nuestro Servicio. Edad media: 61,11 \pm 11,02 , siendo mujeres en el $62,9 \%$ de los casos. Debutaron con hemorragia digestiva en un $40 \%$. La mediana de seguimiento fue de 28 meses (3-133). La mortalidad fue de 54,3\%, con recidiva del 40\%. Variables morfológicas: la localización más frecuente fue gástrica, 51,4\% (18). Existió necrosis tumoral en un 57,1\%, 20. El patrón celular fue fusocelular en un $57,1 \%$, y epitelioide en un $14,3 \%$. El diámetro máximo fue de 9,58 $\pm 6,29$. El índice mitótico por 50 campos de gran aumento fue de 13,44 $\pm 16,08$. En un $51,45 \%, 18$, fueron neoplasias de alto riesgo. Valores inmunohistoquímicos: CD117+, 85,7\%. PDGFRA+, 85,7\%. CD34+, 77,1\%. EGFR+, 62,9\%. S100+, 34,3\%. Actina+, 20\%. Vimentina+, 100\%. p53+, 40\%. ki67+, 10,71 \pm 10,82. La expresión de EGFR no se relacionó con la recidiva y/o mortalidad del enfermo,

\begin{abstract}
Introduction: the epidermal growth factor receptor, EGFR (HER-1), is a tyrosine kinase receptor. EGFR activation plays an important role in increased cell proliferation, angiogenesis, and decreased apoptosis. Our objective was to study EGFR immunoexpression in GIST, as well as its prognostic value.

Patients and method: a retrospective study that included all patients operated on with a histologic diagnosis of GIST at Department of Surgery, Hospital General, Ciudad Real, between 1995 and 2007. Clinical features: age, sex, manifestations, mortality, recurrence. Pathological features: origin, size, tumoral necrosis, mitotic index, cell type. Immunohistochemical features: vimentin, (V9, Dako A/s); smooth muscle actin (HHF-35, Biogenex); CD34 (QBEND/10); S100 (Policlonal Dako A/S), CD117, (c-kit Rabbit, antihuman polyclonal antibody, 1:600); PDGFR-alfa (Rabbit polyclonal antibody, 1:50, Sta. Cruz Biotechnology). Prognostic molecular features: P-53, PAb240 (DakoCytomation) 1:75; Ki-67, clona MIB1 (Dako), 1:120 y (EGFR) pharmDx ${ }^{\mathrm{TM}}$ Dako Autostainer (Dako, Denmark). Malignancy critera: Fletcher's critera.

Results: from 1995 to 2007, 35 GISTs were resected in our Department. Mean age: $61.11 \pm 11.02$, with a female predominance of $62.9 \%$. Initial clinical manifestation included digestive hemorrhage in 40\%. Median follow-up was 28 months (3-133). Mortality was $54.3 \%$, and recurrence rate was $40 \%$. The most frequent origin was the stomach, $51.4 \%$, (18). There was tumor necrosis in $57.1 \%(20)$. There were spindle-like cells in $57.1 \%$, and epithelioid cells in $14.3 \%$. Mean size was $9.58 \pm 6.29$. Mitotic index per 50 high-power fields was $13.44 \pm 16.08 ; 51.45 \%$ (18) were high-risk tumors. Immunohistochemical expression: CD117+, 85.7\%. PDGFRA+, 85.7\%. CD34+, 77.1\%. EGFR+, 62.9\%. S100+, 34.3\%. Actin+, 20\%. Vimentin+, 100\%. p53+, $40 \%$. ki67 $+10.71 \pm 10.82$. There was no correlation between EGFR expression and recurrence and/or mortality, $p=0.156$ and $p=0.332$, respectively. Mitosis index related to mortality, $p$ $=0.02$, and recurrence, $\mathrm{p}=0.013$.
\end{abstract}


$p=0,156$, y $p=0,332$, respectivamente. El índice mitótico se relacionó con la mortalidad del enfermo, $\mathrm{p}=0,02$, y recidiva neoplásica, $p=0,013$.

Conclusión: en nuestra muestra no existió relación entre la inmunotinción de EGFR y el pronóstico del tumor estromal gastrointestinal.

Palabras clave: Tumores estromales gastrointestinales. Pronóstico. EGFR.
Conclusion: in our study there was no relation between EGFR immunohistochemical expression and the prognosis of GIST.

Key words: Gastrointestinal stromal tumors. Prognosis. EGFR.

Padilla D, Menéndez P, García M, Villarejo P, Cubo T, Gambí D, Pardo R, Martín J. Determinación inmunohistoquímica y utilidad pronóstica del receptor del factor de crecimiento epidérmico en los tumores estromales gastrointestinales. Rev Esp Enferm Dig 2008; 100: 752-757.

\section{INTRODUCCIÓN}

Los tumores estromales gastrointestinales (GIST) son las neoplasias mesenquimales de mayor frecuencia del tracto gastrointestinal. Su origen, diferenciación y pronóstico ha sido muy debatido e históricamente han sido considerados como leiomiomas o leiomiosarcomas. Sin embargo, los GIST difieren de leiomiomas y schwannomas, histológica y estructuralmente. Presentan positividad débil y focal para marcadores inmunohistoquímicos musculares como son la desmina y actina muscular, y ausencia de inmunorreactividad para el marcador neural S-100 $(1,2)$. La identificación en la mayoría de los GIST, de mutaciones en el gen KIT, el cual codifica un receptor con actividad tirosina quinasa nos permite caracterizar esta entidad patológica en relación a otras neoplasias (3-6). Dado que es difícil conocer el pronóstico definitivo de estas neoplasias en nuestro trabajo intentamos conocer su comportamiento de acuerdo a un perfil inmunohistoquímico en el que incluimos el receptor del factor de crecimiento epidérmico (EGFR). Nuestro objetivo será conocer el valor pronóstico de la determinación inmunohistoquímica de EGFR en los GIST, y mostrar las posibles alternativas terapéuticas derivadas de esta inmunotinción (1-7).

\section{PACIENTES Y MÉTODO}

Estudio retrospectivo de todos los tumores estromales gastrointestinales intervenidos quirúrgicamente con carácter curativo en el Servicio de Cirugía General del Hospital General de Ciudad Real, entre los años 1995 y 2007.

\section{Variables}

Clínicas: edad, sexo, clínica, mortalidad, recidiva. $P a-$ tológicas: a) macroscópicas: localización, diámetro; b) microscópicas: necrosis tumoral, índice mitótico; y c) in- munohistoquímicas: se excluyeron las neoplasias que presentaron diferenciación muscular o neural y que se caracterizaron por la tinción para anticuerpo monoclonal frente a actina específica de músculo liso y S-100 respectivamente, siendo la tinción negativa para CD34. El estudio inmunohistoquímico se realizó utilizando anticuerpos frente a:

-CD117:CD117, c-kit Rabbit anti-human polyclonal antibody. Dako Anticuerpo policlonal, concentrado.1:600. PDGFR-alfa: PDGFR-alfa (C-20) Rabbit polyclonal antibody. Santa Cruz Biotechnology. Anticuerpo policlonal concentrado. 1:50. Vimentina (V9, Dako A/s). Actina: actina del músculo liso (HHF-35, Biogenex). CD34 (QBEND/10). S100 (Policlonal Dako A/S. P53: Se utilizó para P-53, proteína anómala nuclear tras inactivación del gen supresor p53, anticuerpo monoclonal PAb240 (DakoCytomation), 1:75. Ki67: para Ki-67, marcador de proliferación celular, se utilizó la clona MIB1 (Dako), 1:120.

-EGFR, para el estudio de EGFR, de los bloques de parafina se realizaron cortes de $3 \mu \mathrm{M}$ para tinción con hematoxilina y eosina e inmunohistoquímica. El estudio de la expresión inmunohistoquímica del receptor del factor de crecimiento epidérmico (EGFR) se realizó de forma protocolizada mediante el EGFR pharmDx ${ }^{\mathrm{TM}}$ Kit para EGFR (HER1) en células que expresaron el EGFR. El kit EGFR pharmDx IHC contiene reactivos necesarios para completar un procedimiento de tinción IHQ de muestras incluidas en parafina, fijadas de la forma habitual. Tras la incubación con el anticuerpo monoclonal primario antiproteína EGFR humana, clon 2-18C9, utilizamos un reactivo de visualización listo para usar basado en la tecnología del dextrano, eliminando la necesidad de la aplicación secuencial de conjugado de peroxidasa y anticuerpo de enlace. La conversión enzimática del cromógeno añadido posteriormente produce la formación de un producto de la reacción visible en la zona del antígeno. La muestra se contratiñó con hematoxilina y se colocó un cubreobjetos. Los resultados se interpretaron con microscopio óptico (Fig. 1). 


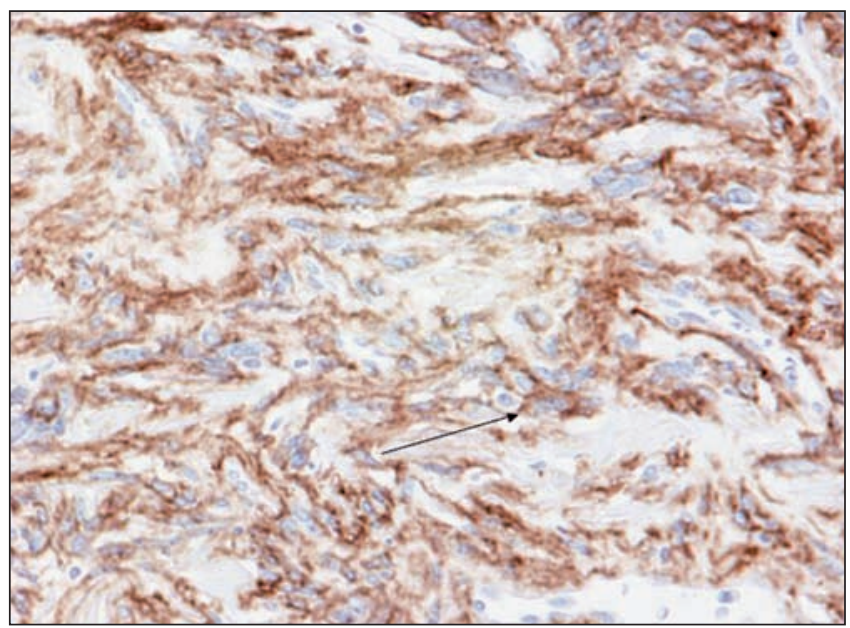

Fig. 1. Inmunotinción positiva de EGFR, 400x.

\section{Criterios de malignidad}

Utilizamos los criterios de Fletcher y cols. $(4,6)$, para conocer el riesgo de malignidad de estos tumores, considerando los valores índice mitótico y tamaño tumoral considerando los estadios tumorales siguientes: riesgo muy bajo, $<2 \mathrm{~cm}$ y $<5$ mitosis/50 campos de gran aumento (cga). Riesgo bajo, $2-5 \mathrm{~cm}$ y $<5$ mitosis/50 cga. Riesgo intermedio, $5-10 \mathrm{~cm} \mathrm{y}<5 \mathrm{mit} / 50 \mathrm{cga}$, $>5 \mathrm{~cm}$ y $>5 \mathrm{mit} / 50 \mathrm{cga}$. Riesgo alto $>5 \mathrm{~cm} \mathrm{y}>5$ mit $/ 50 \mathrm{cga},>10 \mathrm{~cm}$ y cualquier índice mitótico, cualquier tamaño y $>10 \mathrm{mit} / 50 \mathrm{cga}$.

\section{Análisis estadístico}

Estudio retrospectivo de los tumores estromales gastrointestinales intervenidos quirúrgicamente en nuestro Servicio, desde el año 1995 hasta 2007. Utilizamos la prueba de Chi cuadrado para variables cualitativas. Utilizamos las pruebas no paramétricas de Mann WhitneyWilcoxon para dos categorías, y Kruskal-Wallis para más de dos categorías. El análisis de recidiva y mortalidad se realizó mediante el modelo de regresión de Cox y el método de Kaplan Meier, comparando con prueba de rangos logarítmicos para valores dicotómicos. Utilizamos en variables cuantitativas el valor medio como punto de corte. El análisis se realizó mediante el programa SPSS 11.0 para Windows (SPSS Inc., Chicago, IL, EE. UU.).

\section{RESULTADOS}

Entre 1995 y 2007, 35 enfermos con diagnóstico de tumor estromal gastrointestinal (GIST), fueron intervenidos en el Servicio de Cirugía General y Aparato Digestivo del Hospital General de Ciudad Real, con intención curativa. La edad media fue de 61,11 $\pm 11,02$ (33-82), siendo mujeres en un $62,9 \%$ de los casos. En un $40 \%$ debutaron con hemorragia digestiva. La mediana de seguimiento fue de 28 meses (3-133), con supervivencia media de 39 meses. Diecinueve enfermos fallecieron $(54,3 \%)$ y existió recidiva en 14 ocasiones -carcinomatosis peritoneal (4) y metastatización hepática (10)-. Se instauró tratamiento con mesilato de imatinib en tres enfermos de acuerdo al protocolo de actuación del servicio de oncología de nuestro hospital.

- Variables morfológicas. La localización más frecuente fue gástrica, 51,4\% (18). Existió necrosis tumoral en un $57,1 \%$ (20). El patrón celular fue fusocelular en un $57,1 \%$, y epiteloide en un $14,3 \%$. El diámetro máximo fue de 9,58 $\pm 6,29$. El índice mitótico por 50 campos de gran aumento fue de 13,44 $\pm 16,08$. En un $51,45 \%$ (18), fueron neoplasias de alto riesgo.

-Variables inmunohistoquímicas. CD117+ (85,7\%). PDGFRA+ $(85,7 \%)$. CD34+ (77,1\%). EGFR $(62,9 \%)$. S100+ $(34,3 \%)$. Actina+ (20\%). Vimentina+ (100\%). P53+ (40\%). Ki67+, 10,71 $\pm 10,82$ (\% positividad).

$\mathrm{El}$ análisis de recidiva y mortalidad del enfermo en relación a la expresión de EGFR, no mostró datos significativos, $\mathrm{p}=0,156$ y $\mathrm{p}=0,332$, respectivamente. Tampoco existió correlación entre la inmunodeterminación de EGFR y el resto de variables. Sí existió correlación entre el tamaño tumoral y el índice mitótico, $\mathrm{p}=0,01$, y entre el índice mitótico y Ki67, p < 0,001. El índice de mitosis celular se relacionó de forma independiente con la mortalidad del enfermo, $p=0,020$, y recidiva, $p=0,013$. Realizamos caracterización clínica, patológica e inmunohistoquímica con mayor especificidad en relación a la localización, y definición como alto grado de malignidad según Fletcher y cols. $(4,6)$.

- Localización intestinal. La edad media fue 59,81 \pm 10,52 . Un $75,5 \%$ fueron mujeres. En un $37,5 \%$ se presentaron con hemorragia digestiva. Existió mortalidad en un $56,3 \%$ y recidiva en un $50 \%$. La localización fue duodenal, 43,8\% (7), yeyuno-íleon, 43,4\% (7), intestino grueso, $12,5 \%$ (2). El patrón celular fue fusocelular $(56,3 \%)$. El diámetro máximo fue $9,07 \pm 6,35 \mathrm{~cm}$. Existió necrosis tumoral en un $68,8 \%$. El índice mitótico fue de 18,93 \pm 21,51/cga. Variables inmunohistoquímicas: CD117+ $(81,3 \%)$. PDGFRA+ $(81,3 \%) \cdot$ CD34+ $(62,5 \%)$. EGFR+ $(62,5 \%) . \mathrm{S} 100+(56,3 \%)$. Actina+ $(18,8 \%)$. Vimentina+ $(100 \%) . \mathrm{P} 53+(50 \%) . \mathrm{Ki} 67+, 12,88 \pm 11,73$.

-Localización gástrica. La edad media fue 62,61 \pm 11,80 . Un 50\% fueron varones. En un 44,4\% se presentaron con hemorragia digestiva. Existió mortalidad en un $50 \%$, y recidiva en un $27,8 \%$. El patrón fue fusocelular en un 55,6\%. El diámetro máximo fue 9,07 $\pm 5,01$ $\mathrm{cm}$. Existió necrosis tumoral en un $44,4 \%$. El índice mitótico fue $8,06 \pm 7,15 / \mathrm{cga}$. Variables inmunohistoquímicas: CD117+ (88,9\%). PDGFRA $+(83,3 \%)$. CD34+ $(88,9 \%)$. EGFR+ $(66,7 \%) \cdot \mathrm{S} 100+(16,7 \%)$. Actinat $(16,7 \%)$. Vimentinat $(100 \%)$. P53+ $(27,8 \%)$. $\mathrm{Ki} 67+, 7,17 \pm 6,68$. 
- GIST de alto grado de malignidad. La edad media fue $60,61 \pm 11,62$. Un 55,6\% fueron mujeres. En un $44,4 \%$ se presentaron como hemorragia digestiva. Existió mortalidad en un $77,8 \%$ y recidiva en un $66,7 \%$. El patrón fue fusocelular en un $50 \%$ de las ocasiones. El diámetro máximo fue de 13,19 $\pm 6,66 \mathrm{~cm}$. Existió necrosis tumoral en un $83,3 \%$. El índice mitótico fue de 22,44 \pm 17,62/cga. Variables inmunohistoquímicas: CD117+ (83,3\%). PDGFRA+ (77,8\%). CD34+ (88,9\%). EGFR+ $(61,1 \%) . \mathrm{S} 100+(22,2 \%)$. Actina+ $(27,8 \%)$. Vimentina+ $(100 \%) . \mathrm{P} 53+(55,6 \%) . \mathrm{ki67}+, 15,78 \pm 12,55$ (Tabla I).

Tabla I. Patrón inmunohistoquímico en relación a localización y alto riesgo

\begin{tabular}{lccc}
\hline & $\begin{array}{c}\text { GIST intestinales } \\
+\end{array}$ & $\begin{array}{c}\text { GIST gástricos } \\
+\end{array}$ & $\begin{array}{c}\text { GIST de alto riesgo } \\
+\end{array}$ \\
\hline CD117 & $81,3 \%$ & $88,9 \%$ & $83,3 \%$ \\
PDGFR & $81,3 \%$ & $83,3 \%$ & $77,8 \%$ \\
CD34 & $62,5 \%$ & $88,9 \%$ & $88,9 \%$ \\
EGFR & $62,5 \%$ & $66,7 \%$ & $61,1 \%$ \\
S100 & $56,3 \%$ & $16,7 \%$ & $22,2 \%$ \\
Actina & $18,8 \%$ & $16,7 \%$ & $27,8 \%$ \\
Vicentina & $100 \%$ & $100 \%$ & $100 \%$ \\
P53 & $50 \%$ & $27,8 \%$ & $55,6 \%$
\end{tabular}

También realizamos un perfil clínico, patológico e inmunohistoquímico de aquellos enfermos con peor pronóstico y recidiva de la enfermedad, y aquellos enfermos con un supuesto mejor pronóstico (Tabla II).
- Enfermos con recidiva de la enfermedad. La edad media fue de $60,79 \pm 9,84$. Un $50 \%$ fueron mujeres. En un $57,1 \%$ la manifestación clínica fue existencia de masa abdominal. Existió mortalidad del 92,9\%. El patrón celular fue fusocelular en un $57,1 \%$. El diámetro máximo fue de 12,42 $\pm 7,47$. Existió necrosis tumoral en un 85,75. El índice mitótico fue de 12,42 7,40. Variables inmunohistoquímicas: CD117+ (92,9\%). PDGFRA+ (78,6\%). CD34+ (92,9\%). EGFR+ (57,1\%). S100+ (28,6\%). Actina+ $(28,6 \%)$. Vimentina+ (100\%). p53+ (64,3\%). Ki67, $16,71 \pm 13,00$.

- Enfermos con muy bajo, bajo y mediano riesgo. La edad media fue de 61,65 $\pm 10,68$ años (47-82). El 70,6\% fueron mujeres. Existió hemorragia digestiva en un 35,35. La mortalidad fue de 29,45. El patrón fusocelular existió en un 64,7\%. El diámetro máximo fue de 5,76 \pm $2,59 \mathrm{~cm}$. Existió necrosis tumoral en un 29,4\%. El índice mitótico fue de 3,31 $\pm 1,26$. Variables inmunohistoquímicas: CD117+ (88,2\%). PDGFRA+ (82,4\%).CD34+ $(64,7 \%) \cdot \mathrm{EGFR}+(64,7 \%) . \mathrm{S} 100+(47,1 \%)$. Actina+ $(47,1 \%)$. Vimentina+ (100\%). p53+ (23,5\%). ki67, 5,35 4,70. No existió relación entre la expresión de EGFR y mortalidad del enfermo $(\mathrm{p}=0,880)$, y recidiva de la enfermedad ( $p=0,293)$.

\section{DISCUSIÓN}

El EGFR (HER-1) es un receptor tirosina quinasa cuya activación permite un incremento de la proliferación celular, angiogénesis, metástasis y descenso de la apoptosis celular. La sobreexpresión de EGFR se debe a una muta-

Tabla II. Perfil clínico, patológico e inmunohistoquímico de enfermos con recidiva de la enfermedad, enfermos con GIST de alto riesgo y enfermos con GIST de muy bajo, bajo y mediano riesgo

\begin{tabular}{|c|c|c|c|}
\hline & Enfermos con recidiva & $\begin{array}{l}\text { GIST con muy bajo, } \\
\text { bajo y mediano riesgo }\end{array}$ & GIST de alto riesgo \\
\hline $\mathrm{n}$ & 14 & 17 & 18 \\
\hline \multicolumn{4}{|l|}{ Edad media (rango) } \\
\hline $\begin{array}{l}\text { (mediana), anos } \\
\text { Sexo }\end{array}$ & $\begin{array}{l}60,79(45-78)(61) \\
\text { Muieres } 50 \%\end{array}$ & $\begin{array}{l}61,65(48-72)(64) \\
\text { Muieres } 70.6 \%\end{array}$ & $\begin{array}{l}60,61(33-78)(61) \\
\text { Muieres } 556 \%\end{array}$ \\
\hline Clínica & Masa abdominal (57 1\%) & Hemerragia (35 3\%) & \\
\hline Mortalidad & (J) & & \\
\hline & $92,9 \%$ & $29,4 \%$ & $77,8 \%$ \\
\hline \multicolumn{4}{|l|}{ Diámetro máximo } \\
\hline Media (rango) (mediana), cm & $12,42(2-27)(12,75)$ & $5,76(2-11)(6)$ & $13,19(5-27)(13,75)$ \\
\hline Necrosis tumoral & $85,7 \%$ & $29,4 \%$ & $83,3 \%$ \\
\hline Índice mitótico & $12,42 \pm 7,47$ & $3,31 \pm 1,26$ & $22,44 \pm 17,82$ \\
\hline CD117+ & $92,9 \%$ & $88,2 \%$ & $83,3 \%$ \\
\hline PDGFRA+ & $78,6 \%$ & $82,4 \%$ & $77,8 \%$ \\
\hline CD34+ & $92,9 \%$ & $64,7 \%$ & $88,9 \%$ \\
\hline EGFR+ & $57,1 \%$ & $64,7 \%$ & $61,1 \%$ \\
\hline S100+ & $28,6 \%$ & $47,1 \%$ & $22,2 \%$ \\
\hline Actina+ & $28,65 \%$ & $11,8 \%$ & $27,8 \%$ \\
\hline Vimentina+ & $100 \%$ & $100 \%$ & $100 \%$ \\
\hline $\mathrm{P} 53+$ & $64,3 \%$ & $23,5 \%$ & $55,6 \%$ \\
\hline Ki67 (\% positividad) & $16,71 \pm 13,00$ & $5,35 \pm 4,70$ & $15,78 \pm 12,55$ \\
\hline
\end{tabular}


ción del gen EGFR localizado en el cromosoma 7p12p22. Esta mutación permite el incremento de la transcripción de EGFR o translación, disminución de la destrucción proteica y sobreexpresión de ligandos que se unen al receptor. La sobreexpresión de EGFR nos permitirá el desarrollo de nuevas y esperanzadoras alternativas terapéuticas. El EGFR se sobreexpresa en neoplasias como el cáncer de pulmón, cáncer de colon, cáncer de ovario, vejiga, y cabeza y cuello.

El receptor de crecimiento del factor de crecimiento epidérmico (EGFR), consiste en una glicoproteína de 170 $\mathrm{Kd}$, con un parte con expresión extracelular, dominio extracelular y una porción intracelular caracterizada por la existencia de actividad tirosina quinasa.

Pertenece a la familia ErbB, que lo forman cuatro receptores con dominios intracelulares similares, con diferencias en la región extracelular: HER-1 (EGFR, ErbB1), HER-2 (neu, ErbB-2), HER-3 (ErbB-3), HER-4 (ErbB-4). Estos receptores regularán mitosis celular, diferenciación y supervivencia celular.

Los cuatro miembros de la familia de receptores ErbB, se sintetizan en la región citoplasmática y posteriormente son transportados a la membrana celular, donde son receptores de al menos siete ligandos, EGF, TGF, betacelulina, epirregulina, neurorregulina, amphirregulina, factor de crecimiento que simula heparina.

Tras la interacción de los ligandos y el EGFR, existe fosforilación de la tirosina permitiendo la activación de la quinasa Janus, Jak2, y la proteína activada mitógeo, MAP. En el caso del receptor EGFR, Erb1, los ligandos específicos son el EGF, TGF, epirregulina, HB-EGF, B-celulina.

Son escasos los autores que han intentado conocer la relación entre el EGFR y los GIST, relacionados en otras neoplasias y asociados con un peor pronóstico de las mismas. Su estudio en sarcomas se ha desarrollado sobre todo en sarcomas sinoviales. En nuestros enfermos la inmunoexpresión sólo existió en el 62,9\% de los casos, siendo del $61,1 \%$ en GIST de alto riesgo de malignidad. Autores como Lopes y cols. (8) encontraron una mayor inmunodetección de EGFR en la muestra estudiada, hasta en un $96 \%$ de los casos, sin embargo no encontraron correlación de esta inmunodetección de EGFR y alteraciones genéticas de EGFR. El porcentaje de inmunotinción se redujo a $84,45 \%$, al incluir en un nuevo estudio a un mayor volumen poblacional (9). Las conclusiones sobre el mecanismo de expresión de EGFR en GIST y su valor pronóstico no quedaron demostrados, destacando que en aquellos enfermos que expresen inmunohistoquímicamente EGFR, podría ser de importancia la posibilidad de tratamiento anti-EGFR (8-12).

En nuestro estudio además de determinar la expresión inmunohistoquímica de EGFR con la idea de desarrollar un perfil inmunohistoquímico característico de los GIST, en relación a la localización y alto riesgo de malignidad, intentamos conocer su valor pronóstico como variable independiente, no encontrando datos que justifiquen su uti- lización como factor pronóstico, aunque nuevos estudios que incluyan un mayor número de enfermos serán necesarios para conocer el verdadero valor pronóstico de la inmunotinción de EGFR en GIST. Tampoco existió relación con el resto de variables anatomopatológicas. El índice mitótico sí se relacionó con la mortalidad del enfermo $(p=0,020)$ y recidiva $(p=0,013)$. El marcador de proliferación celular también se relacionó de forma independiente con la recidiva de la enfermedad $(\mathrm{p}=0,018)$. De igual modo existió correlación entre los valores de ki67 y los dos factores pronóstico universalmente aceptados como son el índice mitótico $(p<0,001)$ y tamaño tumoral $(\mathrm{p}=0,01)$.

El tratamiento de los GIST será quirúrgico, realizando resección radical de la tumoración (R0), incluyendo lesiones metastásicas resecables y reacción en bloque de órganos afectados (13-15). La utilización en situaciones de irresecabilidad o enfermedad a distancia de inhibidores de proteína quinasas como el mesilato de imatinib, ha mostrado resultados esperanzadores. La expresión de EGFR en los GIST, también posibilitaría la introducción de nuevas terapias ante situaciones de resistencia al mesilato de imatinib o bien como tratamiento coadyuvante. Las líneas terapéuticas para conseguir la inhibición del EGFR incluye moléculas que inhiben el dominio intracelular tirosina quinasa, anticuerpos monoclonales que actúan sobre el dominio extracelular del EGFR, y la combinación de inhibidores del factor de crecimiento del endotelio vascular (VEGF) e inhibidores de EGFR (16-26).

\section{BIBLIOGRAFÍA}

1. Damjanov I. Small intestine. En: Damjanov I, Linder J, editors. Anderson's Pathology.10th ed. St. Louis: Mosby-Year Book Inc; 1996. p. 1708-27.

2. Pike AM, Lloyd RV, Appelman HD. Cell markers in gastrointestinal stromal tumors. Human Pathology 1998; 19: 830-4.

3. Miettinen M, Lasota J. Gastrointestinal stromal tumors. Review on morphology, molecular pathology, and differential diagnosis. Arch Pathol Lab Med 2006; 130: 1466-78.

4. Fletcher C, Berman J, Corless C, Gorstein F, Lasota J, Longley BJ, et al. Diagnosis of gastrointestinal stromal tumors. A consensus approach. Hum Pathol 2002; 33: 459-65.

5. Poveda A, Maurel J, Martín J, Artigas V, Casado A, Cervera J, et al. Guía de práctica clínica en los tumores estromales gastrointestinales. Cir Esp 2005; 78: 1-13.

6. Fletcher C, Berman J, Corless C, Gorstein F, Lasota J, Longley BJ, et al. Diagnosis of gastrointestinal stromal tumors: a consencus approach. In J Surg Pathol 2002; 10: 81-9.

7. Suster S. Gastrointestinal stromal tumors. Seminars in diagnostic pathology 1996; 13: 297-313.

8. Lopes LF, Bacchi CL. EGFR and gastrointestinal stromal tumor: an immunohistochemical and Fish study of 82 cases. Modern Pathology 2007; 20: 990-4.

9. Lopes LF, Ojopi LB, Bacchi CE. Gastrointestinal stromal tumor in Brazil: clinicopathology, immunohistochemistry, and molecular genetics of 513 cases. Pathol Int 2008; 58: 344-52.

10. Cai YC, Jiang Z, Vittimberga F, Xu X, Savas L, Woda B, et al. Expression of transforming growth factor and epidermal growth factor receptor in gastrointestinal stromal tumours. Virchows Arch 1999; $435 ; 112-5$.

11. Lanzafame S, Minutolo V, Caltabiano R, Munutolo O, Marino B, Gangliano $\mathrm{G}$, et al. About a case of GIST ocurring during pregnancy 
with immunohistochemical expresion of epidermal growth factor receptor and progesterona receptor. Pathology 2006; 202: 119-23.

12. Wang X, Zhang S, MacLennan GT, Eble JN, Lopez-Beltran A, Yang $\mathrm{X}$, et al. Epidermal growth factor receptor protein expression and gene amplification in small cell carcinoma of the urinary bladder. Clin Cancer Res 2007; 13: 953-7.

13. Sanchez Hidalgo JM, Muñoz Casares FC, Rufián Peña S, Naranjo Torres A, Ciria Bru R, Briceño Delgado J, et al. Tumores del estroma gastrointestinal (GIST): factores pronósticos de supervivencia tras citorreducción R0. Rev Esp Enferm Dig 2007; 99: 703-8.

14. Fernández Salazar LI, Alvárez Gago T, Sanz Rubiales A, Velayos Jiménez B, Aller de la Fuente R, González Hernández JM. Tumores del estroma gastrointestinal (GIST): aspectos clínicos. Rev Esp Enferm Dig 2007; 99: 19-24.

15. Pardo Martínez C, Mayol Martínez J, Hernández Pérez C, Álvarez Fernández-Represa J. Gastric stromal tumors: clinical presentation and surgical options. Rev Esp Enferm Dig 2004; 96: 578-83.

16. Fernández A, Aparicio J. Imatinib y tumor del estroma gastrointestinal (GIST): un tratamiento selectivo frente a una diana molecualar. Rev Esp Enferm Dig 2004; 96: 723-9.

17. Shchmelinin I, Sefc L, Necas E. Protein kinase inhibitors. Folia Biologica (Praha) 2006; 52: 137-48

18. Frolov A, Chahwan S, Ochs M, Arnoletti JP, Pan ZZ, Favorova O, et al. Response markers and the molecular mechanisms of action of Gleevec in gastrointestinal stromal tumors. Molecular cancer therapeutics 2003; 2: 699-709.
19. Blay JY, Le Cesne A, Ray-Coquard I, Bui B, Duffaud F, Debaldo C, et al. Prospective multicentric randomized phase III study of imatinib in patines with advanced gastrointestinal stromal tumors comparing interruption versus continuation of treatment beyond 1 year: the French Sarcoma Group. J Clin Oncol 2007; 25: 1107-13.

20. Samelis GF, Ekmektzzoglou KA, Zografos GC. Gastrointestinal stromal tumours: clinical overwiew, surgery and recent advances in imatinib mesylate therapy. EJSO 2007; 33: 942-50.

21. Croom KF, Perry CM. Imatinib mesylate: in the treatment of gastrointestinal stromal tumours. Drugs 2003; 63: 513-22.

22. Grandis JR, Sok JC. Signaling through the epidermal growth factor receptor during the development of malignancy. Pharmacology and Therapeutics 2004; 102: 37-46.

23. Harari PM. Epidermal growth factor receptor inhibition strategies in oncology. Endocrine-Related Cancer 2004; 11: 689-708.

24. Baselga J, Arteaga CL. Clinical update and emerging trends in epidermal growth factor receptor targeting in cancer. J C Oncol 2005; 23: $2445-59$.

25. Normanno N, Gullick WJ. Epidermal growth factor receptor tyrosine kinase inhibitors and bone metastases: different mechanisms of action for a novel therapeutic application? Endocrine-Related cancer 2006; 13: 3-6.

26. Ciardello F, Troiani T, Bianco R, Orditura M, Morgillo F, Martinelli $\mathrm{E}$, et al. Interaction between the epidermal growth factor receptor (EGFR) and the vascular endotelial growth factor (VEGF) pathways: a rational approach for multi-targetanticancer therapy. Ann Oncol 2006; 17: 109-14. 